\title{
Step by step for Ebola entry
}

In a recent paper, Chandran et al. discovered a role for the cellular cysteine proteases cathepsin B (CatB) and cathepsin L (CatL) in Ebola virus infection.

Ebola virus (EboV) causes outbreaks of rapidly fatal haemorrhagic fever in humans and no effective treatment has been found so far. The EboV surface glycoprotein GP initiates infection by inducing fusion of the virus envelope to the membrane of the target cell. The organization of GP is analogous to the envelope glycoproteins of HIV and influenza virus: it is a trimer of disulphidelinked heterodimers in which the membrane distal subunit GP1 regulates the fusion activity of the transmembrane subunit GP2. From studies of HIV and influenza virus, it has been proposed that EboV infection is triggered by the encounter of GP1 with a host factor such as a membrane receptor or low $\mathrm{pH}$ in endosomes. However, the host factor for EboV GP1 had not been identified.

Chandran et al. observed that a specific inhibitor of the endosomal cysteine protease CatB blocked infection of Vero cells by EboV and by vesicular stomatitis virus (VSV) particles modified to express EboV GP (VSV-GP). In addition, they identified a specific defect in GPdependent infection of cell lines derived from mice with targeted knockout of the gene encoding CatB. Using purified enzymes, they showed that GP1 is a direct target for proteolysis by CatB. Based on these studies, they have proposed a two-step mechanism for activation in which CatB or the closely related enzyme CatL extensively cleaves the C-terminal portion of the $35-\mathrm{kDa}$ GP1 to form an $18-\mathrm{kDa}$ intermediate, and further cleavage of this intermediate by CatB releases GP1 constraints on GP2 and inititates fusion and infection. These studies indicate that proteolysis of $\mathrm{EboV}$ GP1 by CatB and CatL is functionally equivalent to binding of HIV gp120 to CD4/co-receptor. With no effective therapeutics for Ebola haemorrhagic fever, the discovery of a role for CatB and CatL during infection presents an exciting opportunity to test the antiviral efficacy of endosomal cysteine protease inhibitors.

\section{(1) References and links} ORIGINAL RESEARCH PAPER Chandran, $\mathrm{K}$., Sullivan, N. J., Felbor, U., Whelan, S. P. \&

Cunningham J. M. Endosomal proteolysis of the Ebola virus glycoprotein is necessary for infection. Science 14 April 2005 (doi: 10.1126/ science.1110656)

FURTHER READING Dimitrov, D. S. Virus entry: molecular mechanisms and biomedical applications. Nature Rev. Microbiol. 2, 109-122 (2004)

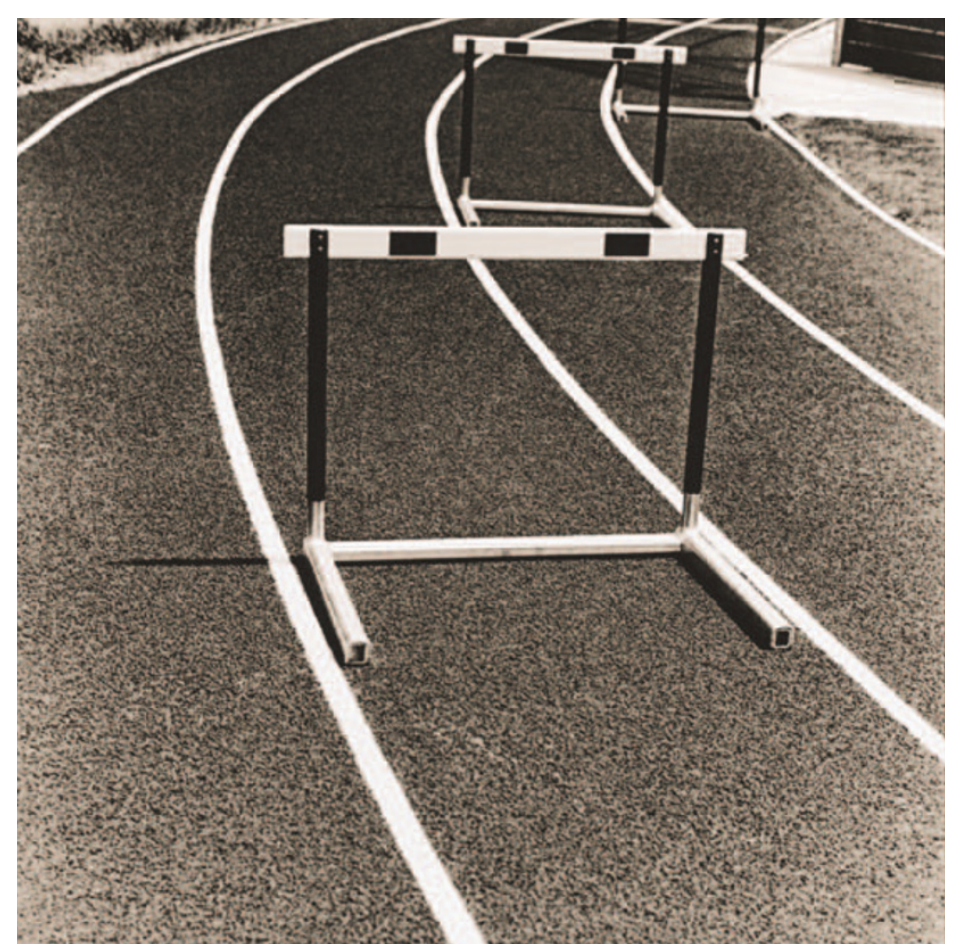

